\title{
THE ROLE OF REGULATION IN THE ORIGIN AND SYNTHETIC MODELLING OF MINIMAL COGNITION
}

\author{
Published in BioSystems \\ The final publication is available at Elsevier via: \\ http://www.sciencedirect.com/science/article/pii/S0303264715001148
}

\begin{abstract}
Leonardo Bich ${ }^{1,2, *}$ and Alvaro Moreno ${ }^{1}$
${ }^{1}$ IAS-Research Center for Life, Mind and Society, Department of Logic and Philosophy of Science, University of the Basque Country (EHU/UPV)

Avenida de Tolosa 70, 20018 Donostia-San Sebastián, Spain
\end{abstract}

${ }^{2}$ Laboratorio de Neurobiologia y Biologia del Conocer (Biology of Cognition Lab), Facultad de Ciencia, Universidad de Chile, Las Encinas 3370, Ñuña, Santiago, Chile

* Corresponding author

email address: leonardo.bich@ehu.es

\begin{abstract}
In this paper we address the question of minimal cognition by investigating the origin of some crucial cognitive properties from the very basic organisation of biological systems. More specifically, we propose a theoretical model of how a system can distinguish between specific features of its interaction with the environment, which is a fundamental requirement for the emergence of minimal forms of cognition. We argue that the appearance of this capacity is grounded in the molecular domain, and originates from basic mechanisms of biological regulation. In doing so, our aim is to provide a theoretical account that can also work as a possible conceptual bridge between Synthetic Biology and Artificial Intelligence. In fact, we argue, Synthetic Biology can contribute to the study of minimal cognition (and therefore to a minimal AI), by providing a privileged approach to the study of these mechanisms by means of artificial systems.
\end{abstract}

Keywords: minimal cognition; regulation; stability; biological autonomy; normativity; Synthetic Biology 


\section{Introduction}

This paper explores the deep connection between biological organisation and cognition at its very roots in basic living systems. The argument that we propose in the following pages pursues two main objectives. In the first place, starting from the framework of biological autonomy, we aim at developing a theoretical account of the origin of some minimal cognitive properties, by analysing the organisational requirements for their realisation: basically, the instantiation of regulatory mechanisms.

One of the essential aspects of cognition, which can be analysed at the basic level, is that cognitive agents should be able to distinguish between some specific features of their interaction with the environment and to act accordingly, in such a way as to maintain their viability ${ }^{1}$. As we shall argue here by developing some of the insights coming from the tradition of research based on the notion of biological autonomy, this implies that they should be able to associate environmental perturbations with internal patterns of self-regulation (Bich and Damiano, 2012a) ${ }^{2}$. All present day living beings have this capacity, let us just think of how bacteria respond adaptively to the composition (and variation in composition) of the environment by means of internal changes, such as the synthesis of different sets of enzymes necessary for metabolising different substances, or by modulating their movement in the environment according to gradients of concentrations, like in the case of chemotaxis (Bich et al., 2015). And it is plausible that more primitive prebiotic selfmaintaining systems had also been capable of maintaining their organisation against environmental perturbations; even though this capacity might have been based on simpler mechanisms ${ }^{3}$.

In the second place, by developing a theoretical account of minimal cognitive properties we aim at providing a bridge between the concepts and languages of Synthetic Biology (SB) and Artificial Intelligence (AI). While AI usually aims at studying and modelling high-level cognitive properties (e.g. at the human level), surprisingly interesting properties for understanding the origin of cognition can be found also in simpler biological systems such as bacteria and invertebrates, and they can provide insights into the functioning of more complex forms of cognition (Bechtel, 2014). Hence, an AI focused on the investigation of cognition at the minimal level requires an approach that is directly linked to biological processes, and this is what SB can provide ${ }^{4}$. The connection between the two disciplines has not been explored exhaustively yet, and the two disciplines still use distinct languages: cognitive for AI and biochemical for SB. Historically, in fact, SB has played an important role in Artificial Life, but not as much with respect to AI, apart from some pioneering

\footnotetext{
${ }^{1}$ By relying on this property, some theoretical approaches to the study of life and cognition have developed the $\mathrm{L}=\mathrm{C}$ thesis according to which cognition is coextensive with life or coincides with the interactive dimension of life (Piaget, 1967; Maturana and Varela, 1973, 1980; Heschl, 1990, Varela et al., 1991: Bitbol and Luisi, 2004, Bourgine and Stewart, 2004). According to other authors, instead, even though those exhibited by minimal living systems are important aspects of cognition, they are not sufficient to define it. According to these latter approaches, it is increased behavioural capacities (Christensen and Hooker, 2000) or a higher degree of organisational complexity, namely a nervous system with its own distinctive norms (Barandiaran and Moreno, 2006; Moreno and Mossio, 2015), which are the primary discriminating dimensions of cognition. We will not address these issues here, as we will limit ourselves to analyse how some specific features related to cognition emerge in biological systems, and not whether or not they can be considered sufficient for full-fledged cognition

${ }^{2}$ For a discussion of the relation between cognition and (homeostatic) regulation in higher organisms with nervous system see for example Damasio, 2003, Ziemke and Lowe, 2009.

${ }^{3}$ It can be argued that proto-mechanisms of regulation, in addition to molecular stability, could have played a role in prebiotic evolution (Bich and Damiano, 2012b)

${ }^{4}$ A clarification is necessary in this respect. We are interested here in that branch of synthetic biology which aims at a better understanding of how living systems work, especially their minimal instances, rather than at engineering organisms that perform specific tasks (for the latter approach see Silver and Way, 2014; Arnold and Meyerowitz, 2014). We refer to that practice of knowledge that, instead of studying living systems by analysing their parts or by formulating predictive models of their behaviours, intends to understand their functioning by actually constructing the object of study (Pfeifer and Scheier, 1999; Damiano et al., 2011, Ruiz-Mirazo and Moreno, 2013), an alternative biological or proto-biological system, and study the properties and behaviours it exhibits.
} 
approaches in bio-chem-ICT (see, e.g. Amos et al, 2011, and Rampioni et al., 2014). The aims, scope and conceptual foundations of this enterprise are still in course of definition and, we argue, a theoretical account of those minimal cognitive properties that can be studied at the level on which SB operates can provide both the missing connections between SB and AI, and a theoretical support for the empirical study of minimal cognition by means of artificial biochemical systems.

On the basis of theoretical considerations on what minimal cognitive properties are and how they originated, we aim at providing a framework for SB-based AI that is distinct in target and goals from existing ones. Focused on developing a dimension of autonomy in artificial systems analogous to that exhibited by living organisms, it aims at a deeper understanding of the origin of minimal cognition in terms of instantiation of regulatory capabilities in artificial systems. In such a scenario the dimension of normativity (Bickhard, 2009; Mossio and Bich, 2014) - how an autonomous system can generate its intrinsic goals and norms, that coincide with its own self-maintenance, rather than having them imposed from outside by the designer - plays a crucial role. Other more engineering-oriented approaches such as those reviewed in Amos et al (2011), instead, are mainly focused input-output relations, and on computing through biochemical systems. The research proposed by Rampioni et al. (2014), on the other hand, shares with our approach a common theoretical framework, that of autonomy, and similar general goals: a better understanding of minimal cognition rather than the development of biochemical tools only. Then it focuses on different issues, even though closely related to those addressed in this paper: the study of bacterial communication and, specifically, of signal transmission between synthetic and living cells namely synthetic cells sending signals to bacterial ones - while we will focus here on the biochemical requirements for the emergence of cognition and for the implementation of cognitivelike properties in synthetic systems.

Our goals, as stated above, are primarily theoretical. In this paper we will mainly focus on how some essential requirements for cognition have appeared. In particular, we will argue that specific mechanisms of internal compensation for perturbations are those responsible for the emergence of a capacity to distinguish between specific features of the interactions with the environment which, otherwise, would constitute only a mere source of noise for the system. In Sections 2 and 3, we will distinguish between two forms of compensation for perturbations: respectively, dynamic stability and adaptive regulation. And we will show how only the second - based on a decoupling between constitutive metabolism and regulatory mechanisms, and on the capacity to produce endogenous interpretations and evaluations of environmental stimuli - enables more complex interactions between an organism and its environment, in which a world of 'meaningful' (i.e. functional for the system) specificities emerges for the system. In Section 4 we will present a comparative case study in which to confront the role of these different compensatory mechanisms in distinct instances of chemotactic behaviour. In Section 5, we will argue in favour of a privileged role for Synthetic Biology in the study of these properties at the very roots of agency and cognition.

\section{Basic self-maintaining metabolic networks: structural stability against environmental noise}

According to the framework based on the notion of biological autonomy (Varela, 1979, Kauffman, 2000; Ruiz-Mirazo and Moreno, 2004) living systems con be characterised as far from equilibrium self-maintaining chemical systems capable of producing their own functional components and physical boundary. In doing so, they maintain themselves as organised unities by promoting the conditions of their own existence through interactions with a changing environment. The idea of biological autonomy (Fig. 1) emphasises (1) the self-referential character of living systems as selfproducing and self-maintaining systems - understood through the notion of organisational closure (Piaget, 1967; Rosen, 1972, 1991; Maturana and Varela, 1973, 1980; Ganti, 1975, 2003; Kauffman, 
2000, Mossio and Moreno, 2010; Montévil and Mossio, 2015) ${ }^{5}$ - and (2) the intrinsically interactive dimension of their organisation: the autonomous organisation cannot exist unless it maintains a continuous coupling with its environment. In such a scenario compensatory mechanisms, by modulating internal processes in relation to environmental changes, constitute a crucial factor in characterising living systems from their most basic instances.

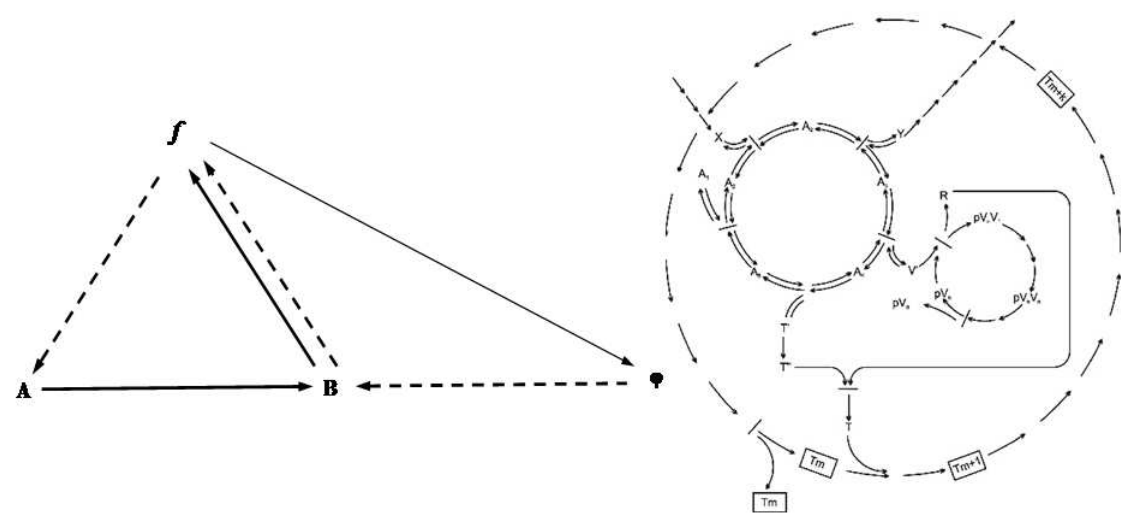

Figure 1. Two models of the organisation of basic autonomous systems: the M/R-System (adapted from Rosen, 1991) and the Chemoton (adapted from Ganti, 2003).

Self-maintaining metabolic systems can implement a variety of qualitatively different response mechanisms, in such a way as to ensure their viability: from simple buffering to the synthesis ondemand of specific sets of enzymes. Let us consider basic responses first. Autonomous systems can respond to environmental changes in the simplest way by means of changes transmitted through the actual network of processes of production of components ${ }^{6}$. This is a behaviour common to basic self-maintaining systems in general. Such systems exhibit what Waddington called 'absorptive buffering' (Waddington, 1968: 14): the network simply absorbs the effects of perturbations through internal reciprocal adjustments between strictly coupled subsystems.

This is what in the literature is referred to as dynamic stability (Rosen, 1970). In biological or protobiological systems it is a property of the self-maintaining network of processes of production, which responds as a whole to perturbations by compensating the displacement of the system from its initial state, thus keeping its dynamics within its range of viability. In this kind of phenomenon the network responses take place through the strict stoichiometrical coupling between the subsystems involved. A variation affecting a given process or subsystem can propagate within the system, and produce the variation of one or several other processes which, in turn, compensate the initial one while the dynamical identity of the system is maintained in the initial attractor, or shifts to a new one. As a result, the system remains stable.

\footnotetext{
${ }^{5}$ There are important differences in how these authors conceive the concept of organisational closure. For a detailed analysis of this question, see Moreno and Mossio (2015).

${ }^{6}$ Let us think of Ganti's chemoton, a model of minimal living system organised as a biochemical clockwork (Ganti, 2003) in which three autocatalytic subsystems - respectively a metabolic cycle, a template subsystem and a compartment - are directly coupled like chemical cogwheels. In such a system any change in one subsystem affects directly the others through supply and demand of metabolites, and can be compensated through changes transmitted through the network. For example, an increase in the amount of nutrients entering the system, after a first rise in metabolic activity, causes an accumulation of the products of the metabolic cycle, thus slowing again the whole dynamics. Yet, other responses are possible, involving also the two other subsystems. For example, after the products of metabolism reach a certain threshold of concentration, determined by the structure and length of the template, the template subsystem consumes them in duplicating itself and as a result activates the production of components of the membrane. In such a way the system, by enlarging the membrane in a way determined by the accumulation of molecules in the system, avoids an increment in the internal pressure and, consequently, maintains its viability through the strict coordination of its three subsystems.
} 
What are the implications of these basic responses for our discussion of the roots of cognition and, more specifically, of the capability of a minimal biological system to handle and endogenously evaluate specific perturbations? The response of the perturbed system is surely dependent on its internal structure, i.e. it is embedded in the organisation of its self-maintaining metabolism. Therefore, the interaction with the environment cannot be simply characterised as a trivial inputoutput relation. Yet, as we shall argue next, stability mechanisms are too simple to account for the origins of specific, meaningful, interactions. The reason is that from the point of view of the internal operations of the system there is no such thing as an endogenous recognition of a specific change and a consequent activation of a response aimed at handling it.

Admittedly, this type of basic - and probably only prebiotic - organisation could enable associations between perturbations and their final effects on the system: the result of internal chains of adjustments. Hence, the system can indeed be said to establish some 'classes of equivalence' (Rosen, 1978) ${ }^{7}$ with respect to its interactions with the environment. Yet, these correlations are valid only for an external observer who identifies correspondences between environmental variations and the results of the changes that they trigger: specifically, conservation of attractor, change of attractor, disintegration of the system. And, indeed, a designer can use this kind of system as a way to classify perturbations or sequences of perturbations according to its own goals and purposes.

Hence, the crucial point here is that these correlations are not endogenously produced distinctions. They concern the final results of perturbations, whereas they do not affect operationally the way this result is achieved: the way the system handles the perturbations by changing its own internal dynamics. A system compensating for perturbations only on the basis of stability responses does not exhibit an internal capacity to functionally identify specific patterns within the set of perturbations disturbing its organisation, to attribute any specific meaning to them, and to act accordingly. For these reasons, in this basic scenario the environment is still an indistinct background, a source of noise, which exerts some influence on the system. The latter has no capacity to distinguish between specific features of its interactions with the environment, which acts only as source of viable or non-viable perturbations.

In sum, basic self-maintaining metabolic networks, however capable of certain forms of 'adaptive' responses, do not display those capabilities necessary for the emergence of minimal cognition. A more complex organisation is necessary, which harbours mechanisms that go beyond such basic metabolism.

\section{Regulation and the emergence of an endogenously generated 'World'}

What is necessary to overcome the inherent limitations of basic structural stability is the instantiation of a dedicated regulatory subsystem able to endow the system with the capability of acting on its own internal dynamics by modifying them in relation to changing external conditions. However, this is not a trivial question. The realisation of a regulatory subsystem does not simply amount to an addition of a new functional structure or subsystem to the basic self-maintaining metabolic network. Adding an additional node, in fact, would just result in a larger functional network, which would still compensate for perturbations through stability behaviours. Hence, what is required for achieving effective regulatory control is a subsystem 'that is sufficiently independent of the dynamics of the controlled processes, and which can be varied without disrupting these processes, but it is still able to be linked to parts of the mechanism controlled system [(the regulated subsystem)] so as to be able to modulate their operations' (Bechtel, 2007: p 290. See also Christensen, 2007). More specifically, the appearance of a regulatory subsystem implies that the

\footnotetext{
${ }^{7}$ The different sets of interactions that trigger equivalent responses in the system.
} 
organism generates within itself a set of processes dynamically decoupled from the dynamics of its constitutive regime (for more details see Bich et al., 2015) .

Dynamical decoupling between the regulatory $(\mathrm{R})$ and the regulated constitutive $(\mathrm{C})$ subsystems means that $\mathrm{C}$ and $\mathrm{R}$ - even though they are correlated through the system they integrate (the components of $\mathrm{R}$ are produced and maintained by the activity of $\mathrm{C}$; and the activity of $\mathrm{C}$, in turn, is modulated by R) - work at different intrinsic rates. This happens when the operating of the regulatory subsystem is neither specified nor determined directly by the metabolic activity of C: i.e., it is 'stoichiometrically free' from the latter (Griesemer and Szathmáry, 2009). More specifically, the idea at the basis of this form of decoupling is that the activation and operation of the regulatory subsystem ( $\mathrm{R}$ ) is not directly dependent on its concentration (or variation of concentration), that is, on its production by $\mathrm{C}$, even though $\mathrm{C}$ guarantees its presence in the system. On the contrary, the activation of $\mathrm{R}$ is triggered by environmental perturbations, and its operations depend on its internal organisation and on the structure of its functional components. In such a way the regulatory subsystem can work as operationally distinct from $\mathrm{C}$, and can in principle act as a dedicated regulatory controller of $\mathrm{C}$ (Bich et al, 2015).

In this organisational architecture, the functional role of a regulatory subsystem is to modulate the basic constitutive network, by shifting between distinct metabolic regimes available to the system in relation to changes in environmental conditions. It does so in such a way that the new metabolic/constitutive regimes brought forth by regulatory switches should be capable of coping with the new environmental conditions, extending the range of perturbations or stimuli to which the system may respond in a rapid and efficient way, as well as enriching the sphere of dynamic functional behaviours available.

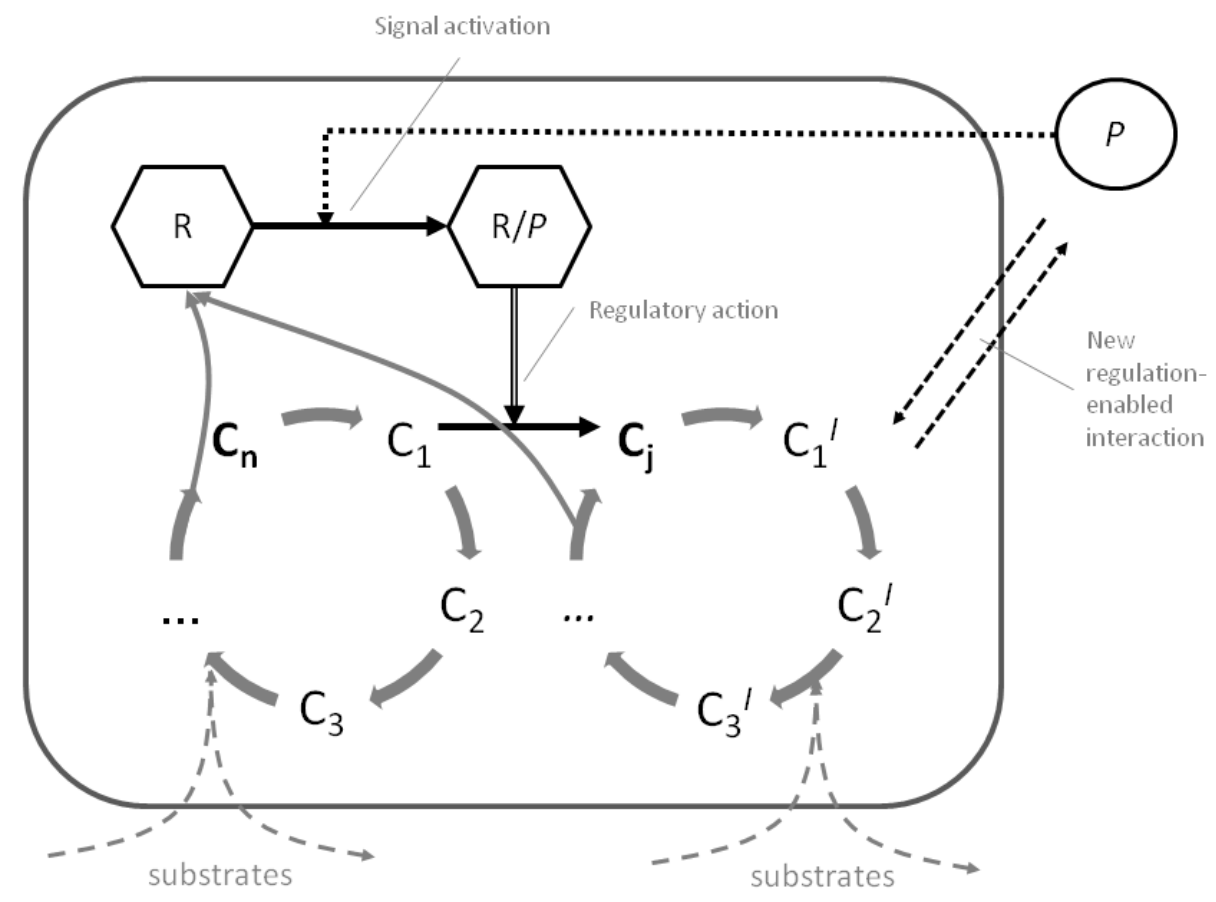

Figure 2. The abstract scheme of basic regulation (Bich et al., 2015)

grey lines: production processes

black lines: processes belonging to the regulatory loop

full arrows: intra-system processes

\footnotetext{
${ }^{8}$ By 'constitutive' we mean here the basic self-maintaining network responsible for the synthesis and maintenance of the constituents of the system.
} 
As represented schematically in Fig. 2 above, and analysed in detail in Bich et al. (2015), the activity of $\mathrm{R}$ unfolds in the following steps. In the absence of perturbations the constitutive regime $\left(\mathrm{C}_{1}-. . \mathrm{C}_{\mathrm{n}}\right)$ interacts viably with the environment. It produces and maintains the regulatory subsystem $(\mathrm{R})$, which does not participate in the production processes or otherwise it would be just a part of a more comprehensive network $\mathrm{C}$. At this stage $\mathrm{R}$ is not operating on $\mathrm{C}$. The first transition takes place with the activation of $\mathrm{R}$. The regulatory subsystem is activated by a specific change/perturbation (or a specific set of changes/perturbations) $\mathrm{P}$ in either internal or external conditions ${ }^{9}$. The subsequent step, the regulatory action, takes place when the activated R/P acts on $\mathrm{C}$, by modulating the activity of the functional components of the latter. In doing so, R/P brings forth a transition to a new different constitutive regime $C^{\prime}\left(C_{1}{ }^{\prime}-\ldots C_{j}{ }^{\prime}\right)^{10}$. Such regulatory action is operationally independent of the activity of $\mathrm{C}$ : it depends only on the organisation of the subsystem $\mathrm{R}$ and on the specific nature of the perturbation $\mathrm{P}$. As a result of it, the new regime $\mathrm{C}^{\prime}$ brought forth by $\mathrm{R} / \mathrm{P}$ is now capable of coping with the environmental variation/perturbation which activated $\mathrm{R}$ to $\mathrm{R} / \mathrm{P}$.

The regulatory subsystem is therefore responsible for mediating the effect of the perturbation $\mathrm{P}$ on the self-maintaining metabolic network $\mathrm{C}$. It does so by modulating $\mathrm{C}$ and integrating the perturbation in a new internal regime capable of coping with it: for example a shift between the metabolisms of different sugars operated by a genetic regulatory subsystem like the lac-operon, which brings forth an adaptive response to variations in the composition of the environment (in this case the presence or absence of specific sugar sources).

Let us consider now the role of regulation in relation to our discussion of the roots of cognition, and compare it to the case of stability. The fundamental difference between the two cases analysed here is that when a mechanism of regulation is at work, the environment is not only a source of indistinguishable perturbations, but also of specific and recognizable ones. The crucial point is that the system endowed with regulatory mechanisms reacts in a very new way: it does things according to what it distinguishes in its interactions with the environment - i.e. what activates the regulatory subsystem. Actually, the 'recognition' in fact is a complex process, since the specific features of the interactions with the environment which are responsible for triggering the regulatory subsystem do not drive directly the response of the system, such as in the case of basic network responses. In fact, the perturbation activates the regulatory subsystem, which in turns modulates the basic constitutive network in such a way as to cope with the specific environmental feature which triggered the regulatory response: the organism eats a new source of food, or secrets chemicals to neutralise a lethal substance, etc. In other words, the fact of recognition is both a consequence of the specificity of the perturbation and of the internal normative organisation of the system.

In such a scenario an environmental perturbation becomes a specific and recognizable interaction because of the nature of the relation it holds with the regulatory subsystem. The regulatory subsystem is sensitive to it in the sense that it endogenously establishes classes of equivalence (Rosen 1978) in its environment with respect to these specific variations: according to how the variations activate $\mathrm{R}$ and trigger the regulatory action. These classes of equivalence do not consist in externally established associations between perturbations and the results of the changes triggered in the system as a whole, as in the previous case. Rather, they are the results of the evaluation

\footnotetext{
${ }^{9}$ It is important to notice that $\mathrm{R}$ is not activated by a variation in its concentration (the amount $\Delta \mathrm{R}$, corresponding to a variation of the production of $\mathrm{R}$ by $\mathrm{C}$ ), since the activation step is not stoichiometrically coupled to the dynamics of $\mathrm{C}$.

${ }^{10}$ By different constitutive regime we mean a basic self-maintaining network (C') that is capable of doing something different than before. The difference between $\mathrm{C}$ and $\mathrm{C}^{\prime}$ can be minimal, for example involving only the presence of one or few enzymes.
} 
(activation of $\mathrm{R}$ plus regulatory action on $\mathrm{C}$ ) operated by the regulatory subsystem, and therefore they achieve an endogenous, operational, significance for the system ${ }^{11}$.

Hence, beyond the debate about whether or not cognition is coextensive with life, it is clear that regulation becomes a necessary condition for the emergence of cognition, if we consider as an essential aspect of cognition the fact that the cogniser should be capable to transform an external influence into an adaptive integration or, in cognitive terms, into a meaningful interpretation (Heschl, 1990). And this fundamental requirement for cognition is realised only by regulation, rather than by basic biological self-production and self-maintenance (i.e. minimal autopoietic systems), as some of the defenders of $\mathrm{L}=\mathrm{C}$ have argued. A system with regulatory capacities, in fact, is able to do things according to what it distinguishes (what the regulatory subsystem is sensitive to). Consequently, the interactions with the environment become more than just a source of indistinct noise, but are converted into a world of endogenously generated (naturalised) significances -- an Umwelt, (von Uexküll, 1926): interactions become functionally 'meaningful' 12 for the system itself, no longer for an external observer only.

In turn, this capacity of sensing specific features of the interaction with the environment enables regulatory-guided actions. The system endowed with these specific regulatory capacities behaves as an adaptive agent because it has the regulatory capacity to discriminate between different environmental conditions and to normatively trigger those actions whose outcomes would become functional for the system. As a matter of fact, virtually all present-day organisms - even the simplest ones - possess the capacity to adapt their actions in accordance with different environmental conditions. They are able to detect potentially deleterious or favourable variations in the environment and to trigger the selection of an adequate functional action within their available repertoire. On the other hand, adaptive agency was preceded by more primitive forms of agency. Probably, cellular proto-metabolisms were homeostatic, in the sense that they compensated for internal and external perturbations by means of network mechanisms integrated and distributed into their constitutive organisation: basically through structural stability.

In the next section we will analyse the differences between these two forms of interaction with the environment (regulatory driven behaviour and stability driven behaviour) by analysing the case of bacterial chemotaxis.

\section{Different forms of tactic behaviour and their significance for the origin of cognition}

Chemotaxis is a form of behavioural adaptive response to changing environmental conditions which typically involves regulatory mechanisms. It consists in the capability to modulate the direction of the swimming movement on the basis of the composition and the gradient of concentration of metabolites in the medium (Eisenbach, 2004; 2007; Wadhams and Armitage, 2004) ${ }^{13}$.

The capability of moving towards or away from a chemical source is not a prerogative of living systems. For example, in the domain of SB there are several relatively simple artificial systems like the well known case of self-propelled oil droplets (Hanczyc and Ikegami, 2010) - which exhibit complex 'self-induced' behaviours by moving directionally within chemical gradients. Yet, even though realizing some positive feedback between internal convection dynamics and movement in the medium, the sustained movement of droplets is qualitatively different from the autonomous

\footnotetext{
${ }^{11}$ Thus, ultimately, it is the whole autonomous organisation of the system what grounds the normative evaluation - that is, the evaluation related to the self-maintenance of the systems.

${ }^{12}$ Here we use the term 'meaning' as synonymous with 'functional for the system' (see Barandiaran, 2008, ch. 5,6). More specifically, in this paper a source of perturbation becomes meaningful when it can distinguished by the system (by R) and such distinction has an operational effect on the system. Through the action of R the system it modulates its own activity (in C) on the basis of this distinction in such a way as to contribute to its own self-maintenance.

${ }^{13}$ See also van Duijin et al. (2006) for a discussion of this phenomenon in the context of the debate about minimal cognition.
} 
self-maintained movement of living cells. The crucial process for the generation of movement takes place at the interface between droplet and environment: the latter plays a direct role in the generation of movement, rather than being 'only' the source of matter, energy, and possible distinctions like it is for living systems ${ }^{14}$. And the direction of droplets taxis is directly controlled by external conditions ( $\mathrm{pH}$ gradients). It is a very interesting case of physical dynamic stability, realised through the direct coupling between the droplet dynamics and its environmental gradients.

While droplets's movement is to an important degree co-determined by the environment, and its direction is governed by the external gradient, this is not the case for bacteria, which are 'intrinsically active' (Bechtel, 2008) due to their capability of self-maintenance. Their movement, in fact, is not only internally generated by the same regime that produces and maintains the system, it is also 'inherently goal-oriented' (Barham, 2012) ${ }^{15}$. Using Kauffman's expression, bacteria are autonomous systems because they 'act on their own behalf' (Kauffman, 2000). Yet, as we shall see below, an inherent activity underlying chemotaxis is not enough to cope with the question discussed in this paper, namely, the emergence of systems endowed with the capacity to detect specific features of their interactions with the environment, to integrate them into their organisation, and to trigger adaptive actions.

In bacterial chemotactic behaviour the control of movement direction is essentially obtained by modulating the rotation of the flagella. There are two possible dynamic or motor states: counterclockwise, which leads to straight movement (running behaviour), and clockwise, which triggers the rotation of the whole bacterium (tumbling behaviour). The default mode is counter-clockwise, allowing the bacterium to move steadily in a given direction. Exploration of the surrounding medium in other directions involves switching back and forth between these two dynamic states.

We can conceive, at least in principle, of a very basic form of chemotaxis in which the modulation of movement neither relies on a dedicated control subsystem, nor depends on the distinction of specific features of their interaction with the environment. As proposed by Goldstein and Soyer (2008) and Egbert et al. (2010), it is possible to hypothesise a mechanism of metabolism-based chemotaxis in which the receptor complex and the signal transduction pathways are absent, while the rotation of flagella is stoichiometrically linked to the metabolic processes. In the model proposed by Egbert et al. (2010) (the third diagram in Fig. 3), it is the concentration of a product of metabolism that determines whether the rotation of the flagella motor is counter-clockwise or clockwise. The result is an interesting case of a stoichiometrical coupling between behaviour and metabolism, which entails a straightforward dependence of the direction of movement on metabolism, while a dedicated regulatory subsystem is absent.

\footnotetext{
${ }^{14}$ The movement of the cell is generated from within, for example by the action of an internally produced flagellum. There are some important considerations to be made regarding the different nature of sustained movement (droplets) with respect to self-maintained autonomous movement (cells):

1) Droplets do not self-maintain like living cells instead do;

2) Droplets' activity (movement) does not depend on getting nutrients (matter and energy) from the environment. They move by consuming the internal oleic anhydride that is already available;

3) There is no normativity in the movement: it does not contribute to the existence and maintenance of the droplet.

4) There is no internal organisational differentiation (no modular units) in the droplets.

It could be still said that the self-sustained movement of the droplets (but not the droplets themselves) can be considered as self maintaining in a minimal sense (although a different sense from the one used in this paper), insofar as the movement is co-driven by internal processes and gradients, and it exhibits a certain degree of robustness.

${ }^{15}$ The intrinsic goal that produces the movement is the maintenance of the organism. For a detailed discussion of intrinsic teleology in biological systems from a perspective based on autonomy, see Mossio and Bich (2014).
} 


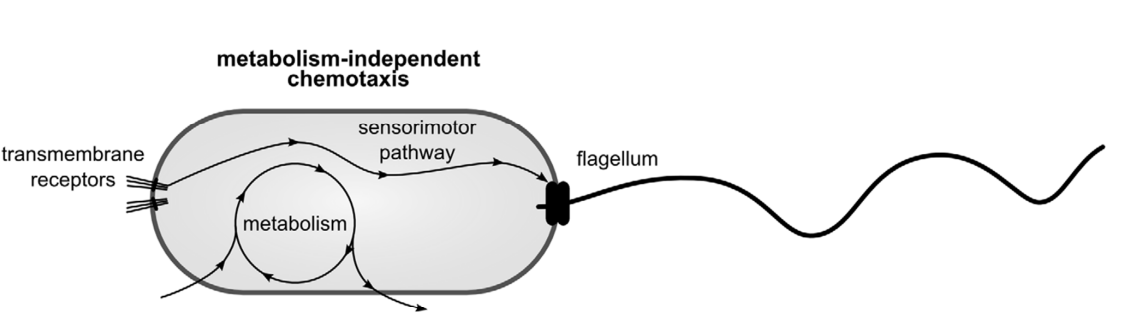

Regulated

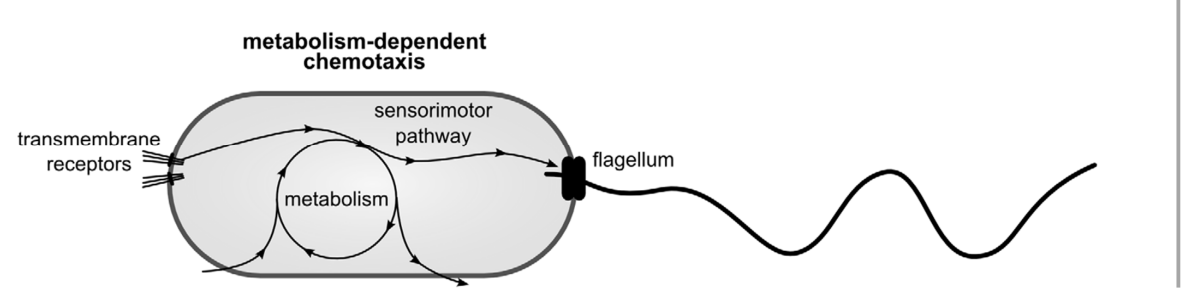

chemotaxis

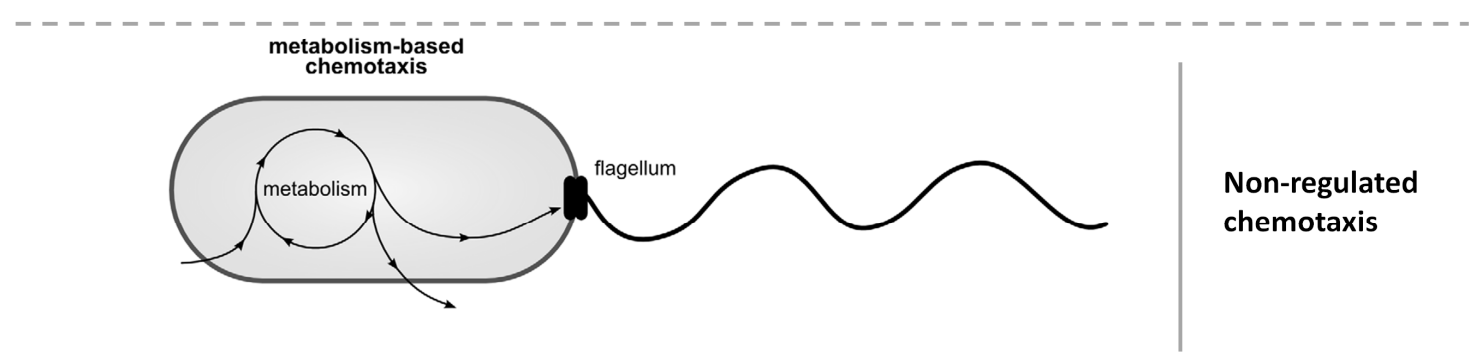

Figure 3. Different forms of regulated and non-regulated chemotaxis (adapted from Egbert et al., 2010)

This case constitutes a complex form of stability-driven taxis, achieved through the coupling of two subsystems, and an indirect feedback through the environment. It is different - and much more complex - from the case of sustained and directional movement in droplet taxis, because movement here is autonomous and depends on the internal organisational differentiation and inherent self-maintaining activity of the cell. In addition, this behaviour is functional in the sense that it contributes to the maintenance of the conditions for the existence of the metabolism and, consequently, of the whole system. Nevertheless, in this example, even though the system is capable of complex viable behaviours, the system responds as a whole network, without distinguishing between specific features of its interaction with the environment, evaluating them and modulating its action accordingly. In this case, as described in Section 2, the environment constitutes only a source of noise which perturbs the metabolism, and the behavioural response is filtered through the latter.

On the contrary, although in a minimal sense, present day bacteria do recognise specific features of their interactions with the environment. The classical model of bacterial chemotaxis, metabolismindependent chemotaxis (top diagram in Fig 3), describes the functioning of the whole mechanism of control of movement as operationally independent from metabolism, even though metabolism must provide the energy for movement and ensure the synthesis and maintenance of the components of the subsystems involved ${ }^{16}$. This chemotactic mechanism involves at least three molecular complexes: (a) a receptor subsystem, which is activated by environmental effectors (chemoattractants or chemorepellants); (b) a flagellum complex, and (c) a group of molecules, which act as a bridge between the two. Usually the latter molecules are activated through noncovalent post-translational modifications, such as cascades of phosphorylations.

\footnotetext{
${ }^{16}$ There are other interesting cases like energy taxis (Alexandre, 2010), which integrates into the regulatory response also an evaluation of the energetic state of metabolism. (middle diagram in Fig. 3).
} 
In particular, the protein $\mathrm{CheY}$ is a central player in the regulatory process. Depending on its different states of activation, it can link to both complexes: receptors and flagellum. In a nonphosphorylated state it binds to the receptor. When the receptor is activated by a change in the effector ${ }^{17}$, CheY is phosphorylated, decreasing its affinity to the receptor and, at the same time, increasing its affinity to the switch protein FliM in the flagellum complex. By detaching from the former and binding to the latter, CheY triggers an alteration of the flagellum rotation from counterclockwise to clockwise and, therefore, determines a change in the swimming movement of the bacterial cell, from running to tumbling. In this manner, the signal transduction pathway (receptor complex plus signal proteins) is able to modulate the movement of the cell.

Stoichiometrically speaking, the activity of the regulatory subsystem (receptor plus bridge/signal proteins) is dynamically decoupled from that of the motor subsystem it modulates, and from metabolism: it is not the synthesis and concentration of CheY and of the other molecules in the receptor subsystem and in the signal transmission pathway that modulate the direction of the rotation of the flagella. The activity of the regulatory subsystem, even if it is materially and energetically supported by metabolism, becomes operationally independent from it, and the modulation it performs on the motor system is not achieved through an increase in the concentration of CheY, but rather by means of cascades of signals and activations. Unlike the previous case of metabolism-based chemotaxis, the regulatory subsystem is activated by a specific set of perturbations (specified by the organisation of the receptors), and can reversibly switch between different states of the flagellum subsystem in relation to them. The regulated behaviour is then able to cope with the perturbations, by allowing changes in the swimming direction. Thereby, it also becomes functional, because it contributes to the maintenance of the system, keeping metabolism within viable conditions.

The distinctive feature of this more complex case of chemotactic behaviour is that the system, thanks to the action of a regulatory subsystem, is capable of modulating its behaviours on the basis of specific interactions with the environment, which acquires a specific operational meaning for the system, in such a way that the latter is able to change its behaviour accordingly. This is made possible by the fact that the regulatory subsystem is decoupled from the others. This decoupling, in fact, introduces a new degree of freedom in the system, one or more new variables in the regulatory subsystem that are not directly dependent on the constitutive regime and therefore can be sensitive to something different than the internal state of the system: in this case a feature of the environment. Such feature acquires significance for the whole system, and the decoupled subsystem achieves a functional role when the effect of its regulatory action, triggered by the perturbation, contributes to the maintenance of the system. Starting from this relatively elementary chemotactic mechanism, then, a variety of more complex tactic behaviours can be implemented (Eisenbach, 2004). What is common to them, is that they cannot be treated in terms of input-output, as if the behaviour of the system were driven by the perturbation. On the contrary, they can be characterised in terms of endogenous generation of adaptive response by focusing on the internal organisation of the system and, especially, of its regulatory subsystems.

In sum, bacterial chemotaxis represents a clear comparative example of how certain capabilities fundamental for the origin of cognition can emerge in minimal living systems, and of how this is only possible through the action of regulatory mechanisms. Even though physical or unregulated biological systems can in principle exhibit similar behavioural phenomenologies, the way changes in environmental conditions are handled and the role they play for the system organisation are radically different. And it is only in the presence of regulation that perturbations acquire a specific significance for the system.

\footnotetext{
${ }^{17}$ It is the organisation of the receptor module that determines which perturbations will trigger an actual regulatory and behavioural response.
} 


\section{Issues related to the investigation of minimal cognitive-like properties by means of artificial systems}

Different approaches can be used to investigate with the aid of artificial systems how these capacities could emerge in minimal biological and, possibly, prebiotic or proto-biological systems: computational, hardware-based and wet biochemical approaches ${ }^{18}$. Yet, these approaches have to face specific issues related to the specificity of the domain in which these properties are realised and of their organisational requirements.

As we have argued in the previous sections, the capability to evaluate perturbations and to act accordingly requires the presence of basic regulatory mechanisms. These mechanisms have specific features, and there arise crucial theoretical issues that have to be taken into consideration for their artificial realisation or for their investigation with the help of artificial model systems.

In the first place, regulatory mechanisms in biological systems are endogenously produced and maintained by the same system that they regulate -- or otherwise we could not speak of biological (self-) regulation, but of externally imposed control --and they functionally contribute to the existence of the same system that produces them. This is a fundamental difference with analogous artificial cybernetic systems, in which parts are put together and just interact without their operations affecting their conditions of existence. In biological systems the generation of regulatory behaviours is not as simple as adding exogenously one or various modules to get a specific or more efficient input-output correlation: it must be developed from within, from a complex network of components and transformation processes functionally linked together to maintain themselves in far from equilibrium conditions; and this involves a more elaborated organisational architecture.

This is the reason why, secondly, one of the organisational aspects of biological regulation - a partial decoupling of the processes within the system - plays a crucial role. In non-chemical artificial systems, in fact, it might be easier to realise or identify a decoupling between regulating and regulated subsystems. In the biochemical domain, though, it is necessary to find ways to free the regulatory subsystem from the stoichiometrical coupling with the constitutive regime, while at the same time having the regulatory subsystem produced from within. Indeed, a synthetic attempt to build and study biological regulatory mechanisms cannot escape the fact that they are realised in the biochemical domain, which has very distinctive properties and fundamental constraints compared to the domains in which computational and hardware models are realised (Rosen, 1991). In particular, the absence of the distinction between software and hardware, or between fixed physical structures and variable operations, does imply looser forms of modularity, as well as transformative interactions. As a consequence, decoupling has to be realised or identified in dynamical rather than physical/structural terms.

A fundamental theoretical issue consists in the fact that we aim at modelling a system to which we can attribute its own purpose and normativity. This internal normativity arises from the dissipative, FFE self-producing/maintaining organisation of living organisms. And that is why we focus on SB rather than on the traditional cybernetic modelling. The goal-oriented dimension of regulation has to be taken into account in its distinctive biological character. As pointed out already by Jonas in his criticism of cybernetics (Jonas, 1966), artificial self-stabilizing or self-regulating systems do not have an intrinsic goal - which, according to the early cyberneticians (Rosenblueth et al, 1943) was just to be identified with the final state - but follow only a purpose specified by an external designer. In living systems, on the other hand, goals are generated within the same system that produces and maintains the regulatory mechanism itself. The goal of artefacts does not coincide with their own existence: it is the case of droplet taxis addressed in Section 4 above, in which movement does not contribute to the conditions of existence of the system. In biological systems as self-maintaining systems, on the other hand, their goal and their own existence are one and the same

\footnotetext{
18 And indeed they are used. The model system proposed by Egbert et al. (2010) for the study of adaptive and evolutionary possibilities opened by metabolism-based chemotaxis is an example of the first approach.
} 
thing (Mossio et al. 2009). Therefore their conditions of existence are also the intrinsic (and naturalised) norms of their own activity: being a dissipative organisation, the system 'must' behave in a specific way, otherwise it would cease to exist. In addition to that, regulated systems do not have just an intrinsic normativity. Regulation adds something by expanding its constitutive normativity: the system in fact not only generates intrinsic norms, but crucially modulates these norms in order to promote its own maintenance. In other words, the regulatory mechanism has, so to speak, an explicit goal, in the sense that it specifies, in each circumstance, how to ensure the maintenance of the system that harbours it.

Given these premises, how can we approach the study of the emergence of minimal cognitive-like properties in artificial systems? Of course, an approach in this direction does not focus on the performance of biologically inspired artificial systems in carrying out some cognitive tasks, but rather aims at a better understanding of the emergence of cognitively relevant capabilities in minimal living systems, considered as contributions to the maintenance of their autonomous organisation. One answer could be, for example, to simulate minimal regulatory behaviours in virtual protocells so as to explore some of their implications and requirements, or even realise simple hardware-based adaptive model systems and observe their behaviours under perturbations. Non-biochemically based artificial systems, though, do not satisfy the requirement for two of the main features of regulation, namely its grounding in metabolic self-production and its intrinsic normativity, which are fundamental in understanding regulation. In this respect the wet (biochemical) domain presents advantages compared to the hardware and computational ones ${ }^{19}$. Simplified proto-cognitive mechanisms can be realised and studied by synthetic tools in the same domain in which they are realised in nature, in a way that makes it possible, at least in principle, to investigate all their distinctive features: from their specific form of decoupling and their realisation from within the system, to their functional contribution to the viability of the system that produces them. Hence, Synthetic Biology can provide modelling tools rooted in metabolic mechanisms, tools that are not directly available to the others domains of Artificial Intelligence ${ }^{20}$. And for these reasons it can constitute an important complement to them.

More specifically, the wet synthetic approach can play a pivotal role in investigating how fundamental properties associated with cognition emerged and acquired a crucial role in early or even proto biological systems. On the basis of the theoretical account developed in the previous sections, the goal of a research programme aimed at studying SB based AI is to realise artificial systems that are endowed with properties that are relevant for minimal cognition: specifically, systems capable to autonomously compensate for perturbations, and which activity is evaluated in terms of self-maintenance and variety of responses, rather than input-out systems, evaluated in terms of performance. This can be done by realising and manipulating biochemical model systems with basic regulatory mechanisms, and by observing their adaptive behaviours in changing environments. In such a way a program based on this theoretical framework can contribute both to the creation of a minimal biochemical artificial 'intelligence' and to an understanding of the role played by cognitive-like properties in the origins and early evolution of life.

Both top-down and bottom-up strategies can be explored in this respect. The former can be implemented by simplifying regulatory mechanisms in actual living organisms down to their still functional basic organisational skeletons. The goal of such an approach would be to identify some fundamental modules for the production of cognitively relevant behaviours. In addition, by manipulating their basic modules, such as their signal receptors, internal circuit organisation or regulatory effectors, it would be possible to observe their different behaviours in changing

\footnotetext{
${ }^{19}$ As it has been argued elsewhere (Rosen, 1991; Moreno and Etxeberria, 2005) the autonomous organisation of organisms cannot be realised but in a chemical domain.

${ }^{20}$ For a discussion of the difference between virtual and biochemical metabolisms see Rosen (1991) and Boden (1999). For a discussion of the importance for AI in general to focus on the organismic roots of agency and cognition see Froese and Ziemke (2009).
} 
environments. These experiments could provide relevant information on how changes induced by manipulations can be compensated or lead to non-viable dead ends, and whether, and under which conditions, they give rise to unexpected emergent functional behaviours.

From the bottom-up point of view, technically the task does not seem an easy one, but some basic insights from the discussion developed in the previous sections can be explored. The idea would be to implement very simple regulatory mechanisms in self-maintaining protocells, and: (a) to study their behaviours in possible pre-biotic environments; or (b) to have these biochemical self-regulated systems interact with one another and study collective proto-cognitive behaviours, as plausible precursors of the behaviour of present day social bacteria ${ }^{21}$. This latter line of investigation could provide information on the possible role played by the collective behaviour of protocells colonies in prebiotic evolution.

One of the main difficulties for this line of research would be to realise dynamical decoupled regulatory mechanisms in such simple systems. A possible way to be explored would be that of stoichiometrical freedom, based at least partially on sequence rather than on concentration, as proposed by Griesemer and Szathmary (2009) in the case of the chemoton. In this respect, some of the best candidates are riboswitches, which can modulate their activity on the basis of interactions with effector molecules in a way analogous to allosteric enzymes, even though they are simpler in terms of structure and composition (Serganov and Nudler, 2013). As demonstrated experimentally by Martini and Mansy (2011), protocells with riboswitches can indeed sense specific molecules and respond to them by triggering gene expression.

\section{Concluding remarks}

How do minimal cognitive properties originate? The answer can be found in the capability of the autonomous organisation of biological systems to compensate for perturbations by distinguishing specific features of their interactions with the environment and respond accordingly.

Most of the theoretical argument developed here has been dedicated to the analysis of the organisational requirements underlying different compensatory responses. We showed how, if we take the capability to make endogenously generated distinctions as a requisite for the emergence of minimal cognition then, contrary to the $\mathrm{L}=\mathrm{C}$ thesis, the basic regime of self-production and selfmaintenance of these systems (a minimal form of autopoiesis) cannot exhibit those minimal cognitively relevant properties, inasmuch as they only respond globally to environmental perturbations by compensating noise. We argued, then, that the presence of decoupled regulatory subsystems marks a fundamental transition in this respect and it constitutes the fundamental requirement for the appearance of minimal cognitive properties. A regulated system, in fact, can evaluate perturbations and respond accordingly, in such a way that some features of the interaction with the environment acquire a meaning for the system, depending on the organisation of its regulatory subsystem. This difference between basic and regulatory responses becomes evident if we compare metabolism-based and metabolism-independent chemotaxis: in the first case the system has no way to evaluate its interactions with the environment, and can only respond to its own internal global state.

By addressing the issue of cognition at the very basic level of biological organisation, the account proposed also bridges some of the gap between biochemical and cognitive concepts by providing an operational characterisation of notions such as distinction, significance, meaning and evaluation. With this organisational account of minimal cognition at hand, we have briefly explored the possibility of establishing a connection between biochemical SB and AI, and we have addressed

\footnotetext{
${ }^{21}$ In this specific case, the objectives of our account converge with those of the approach proposed by Rampioni et al. (2014).
} 
some crucial issues related to the artificial realisation of those minimal regulatory mechanisms at the basis of cognitive-like properties.

Finally, we have come to the conclusion that according to the theoretical account proposed here, the possible contributions of SB to AI could concern the understanding of the mechanism at the origin of some essential properties that are necessary for cognition, and its role in prebiotic evolution or in the early evolution of life. Specifically, they would consist in the implementation of molecular models of basic regulatory mechanisms and of regulation-based interactions with the environment, and in the investigation of the range, variety and complexity of these 'meaningful' interactions considered at the very roots of cognition. In doing so, SB would provide complementary information with respect to more traditional artificial approaches. In particular, by realising its models in the same domain in which proto-cognitive properties are actually instantiated, i.e. the biochemical domain, it can produce not only more plausible models but also unexpected and, therefore, very informative results, due to the systemic effects of biochemical interactions inaccessible to the other approaches.

\section{Acknowledgments}

The authors wish to thank Adele Abrahamsen, William Bechtel, Ingo Brigandt, Karim Bschir, Sara Green, Nicholaos Jones, John Norton, Raphael Scholl, Maria Serban, for the very valuable feedback on an earlier version of this paper. Part of this work was written during LB's stay at the Center for Philosophy of Science of the University of Pittsburgh, funded by the grant FFI2011-25665 of the Spanish Ministry of Economía y Competitividad. The authors acknowledge the grants from the Basque Government (IT 590-13 to AM and LB, and postdoctoral fellowship to LB), from the Spanish Ministry of Economía y Competitividad (FFI2011-25665 to AM and LB), from the Spanish Ministry of Industria y Innovación (BFU2012-39816-C02-02 to AM), and from the Fondo Nacional de Desarrollo Científico y Tecnológico, Chile (Fondecyt Regular-1150052 to LB).

\section{References}

Alexandre, G. (2010). Coupling metabolism and chemotaxis-dependent behaviours by energy taxis receptors. Microbiology, 156, 2283-2293.

Amos, M., Dittrich, P., McCaskill, J., \& Rasmussen, S. (2011). Biological and Chemical Information Technologies. Procedia Computer Science, 7, 56-60.

Arnold, F. H. \& Meyerowitz, J. T. (2014). Evolving with purpose. Nature, 509, 166-167.

Barandiaran, X. (2008). Mental Life. A naturalised approach to the autonomy of cognitive agents. PhD Dissertation. University of the Basque Country (UPV/EHU).

Barandiaran, X. \& Moreno, A. (2006). On What Makes Certain Dynamical Systems Cognitive. A minimally cognitive organisation program. Journal of Adaptive Behavior, 14(2), 171-185.

Barham, J. (2012). Normativity, agency and life. Studies in history and philosophy of biological and biomedical sciences, 43(1), 92-103.

Bechtel, W. (2007). Biological mechanisms: Organised to maintain autonomy. In F. Boogerd, F. Bruggerman, J. H. Hofmeyr, H. V. Westerhoff (eds.). Systems Biology. Philosophical Foundations. Amsterdam: Elsevier, 269-302.

Bechtel, W. (2008). Mental Mechanisms. Philosophical Perspectives on Cognitive Neurosciences. New York: Routledge.

Bechtel, W. (2014). Cognitive biology: Surprising model organisms for cognitive science. Proceedings of the 36th Annual Conference of the Cognitive Science Society. Austin, TX: Cognitive Science Society, 158-163. 
Bich, L. \& Damiano, L. (2012a). Life, Autonomy and Cognition: An Organisational Approach to the Definition of the Universal Properties of Life. Origins of Life and Evolution of Biospheres, 42(5), 389-397.

Bich, L. \& Damiano, L. (2012b). On the emergence of biology from chemistry: a discontinuist perspective from the point of view of stability and regulation. Origins of Life and Evolution of Biospheres, 42(5), 475-482.

Bich, L., Mossio, M., Ruiz-Mirazo, K., \& Moreno, A. (2015). Biological regulation: controlling the system from within. Biology \& Philosophy.

Bickhard, M. (2009). The biological foundations of cognitive sciences. New Ideas in Psychology, 27(1), 75-84.

Bitbol, M. \& Luisi, P. L. (2004). Autopoiesis with or without cognition: Defining life at its edge. Journal of the Royal Society Interface, 1 (1), 99-107.

Boden, M. (1999). Is Metabolism Necessary? The British Journal for the Philosophy of Science, 50, 231-248.

Bourgine, P., \& Stewart, J. (2004). Autopoiesis and cognition. Artificial Life, 10 (3), 327-345.

Christensen W (2007) The evolutionary origins of volition. In: Ross D, Spurret D, Kincaid H, Stephens G L (eds.) Distributed cognition and the will: individual volition and social context. MIT Press, Cambridge MA, pp 255-287.

Christensen, W.D. \& Hooker, C.A. (2000). An interactivist-constructivist approach to intelligence: self-directed anticipative learning. Philosophical Psychology, 13, 5-45

Damasio, AR (2003). Looking for Spinoza: Joy, sorrow and the feeling brain. Orlando, FL: Harcourt.

Damiano, L., Hiolle, A. \& Cañamero, L. (2011), Grounding Synthetic Knowledge. In Lenaerts T., M. Giacobini M., H. Bersini H., P. Bourgine P., M. Dorigo M. and R. Doursat R. (eds), Advances in Artificial Life, ECAL 2011, Cambridge MA: MIT Press, 200- 207.

Egbert, M., Barandiaran, X., Di Paolo, E. (2010). A Minimal Model of Metabolism-Based Chemotaxis. PLoS Computational Biology, 6(2), e1001004.

Eisenbach, M. (2004). Chemotaxis. London: Imperial College Press.

Eisenbach, M. (2007). A Hitchhiker's Guide through Advances and Conceptual Changes in Chemotaxis. Journal of Cellular Physiology. 213, 574-580.

Froese, T. \& Ziemke, (2009). Enactive artificial intelligence: Investigating the systemic organisation of life and mind. Artificial Intelligence, 173(3-4), 466-500.

Ganti, T. (1975). Organisation of chemical reactions into dividing and metabolizing units: the chemotons, BioSystems, 7, 189-195.

Ganti, T. (2003). Chemoton Theory (2 vols.). New York: Kluwer Academic/Plenum Publisher.

Goldstein, R. A. \& Soyer, O. S. (2008). Evolution of taxis responses in virtual bacteria: nonadaptive dynamics. PLoS Computational Biology, 4, e10000084.

Griesemer, J. \& Szathmáry, E. (2009). Ganti's Chemoton model and life criteria. In S. Rasmussen, M. Bedau, L. Chen, D. Deamer, D. C. Krakauer, N. H. Packard \& P. F. Stadler (eds.). Protocells. Bridging Nonliving and Living Matter. Cambridge MA: MIT Press, 481-513.

Hanczyc, M. \& Ikegami, T. (2010). Chemical Basis for Minimal Cognition. Artificial Life, 16, 233243.

Heschl, A. (1990). L=C A simple equation with astonishing consequences. Journal of Theoretical Biology. 145, 13-40. 
Jonas, H. (1966). The phenomenon of life: Towards a philosophical biology. New York: Harper and Row.

Kauffman, S. (2000). Investigations. New York: Oxford University Press.

Martini, L., \& Mansy, S. (2011). Cell-like systems with riboswitch controlled gene expression. Chemical Communications, 47, 10734-10736.

Maturana, H. \& Varela, F. J. (1973), De Máquinas y Seres Vivos: Una teoría sobre la organización biológica, Santiago: Editorial Universitaria (In Maturana, H \& Varela, F. J. (1980). Autopoiesis and Cognition. The Realisation of the Living. Dordrecht: Reidel Publishing).

Montévil, M. \& Mossio, M. (2015). Biological organisation as closure of constraints. Journal of Theoretical Biology, 372, 179-191.

Moreno, A. \& Etxeberria, A. (2005). Agency in natural and artificial systems. Artificial Life, 11(12), 161-176.

Moreno, A. \& Mossio, M. (2015). Biological autonomy: a philosophical and theoretical enquiry. New York: Springer.

Mossio, M. \& Bich, L. (2014). What makes biological organisation teleological? Synthese.

Mossio, M. \& Moreno, A. (2010). Organisational closure in biological organisms. History and Philosophy of the Life Sciences, 32(2-3), 269-288.

Mossio, M., Saborido C. \& Moreno, A. (2009). An Organisational Account of Biological Functions. British Journal for the Philosophy of Science, 60,813-841.

Pfeifer R, \& Scheier C (1999). Understanding Intelligence. Cambridge, MA: MIT Press.

Piaget, J. (1967). Biologie et connaissance. Paris: Gallimard (Biology and Knowledge. Chicago: University of Chicago Press, 1971).

Rampioni, G., Mavelli, F., Damiano, L., D’Angelo, F., Messina, M., Leoni, L., \& Stano, P. (2014). A synthetic biology approach to bio-chem-ICT: first moves towards chemical communication between synthetic and natural cells. Natural Computing, 13, 333-349.

Rosen, R. (1970). Dynamical System Theory in Biology. Stability Theory and Its Applications. New York: John Wiley \& Sons.

Rosen, R. (1972). Some relational cell models: the metabolism-repair systems. In R. Rosen (ed.), Foundations of Mathematical Biology. New York: Academic Press, vol. II, 217-253.

Rosen, R. (1978). Fundamentals of Measurement and Representation of Natural Systems. New York: North Holland.

Rosen, R. (1991). Life Itself. New York: Columbia University Press.

Rosenblueth, A., Wiener, N. \& Bigelow, J. (1943). Behaviour, Purpose and Teleology. Philosophy of Science, 10 (1), 18-24.

Ruiz-Mirazo, K. \& Moreno, A. (2004). Basic Autonomy as a Fundamental Step in the Synthesis of Life. Artificial Life, 10, 235-259.

Ruiz-Mirazo, K. \& Moreno, A. (2013). Synthetic Biology: Challenging Life in Order to Grasp, Use, or Extend It. Biological Theory. 8(4), 376-382.

Serganov, A. \& Nudler, E. (2013). A Decade of Riboswitches. Cell, 152, 17-24.

Silver, A. P., \& Way, J. C. (2014). Rationalizing nature. Nature, 509, 166.

Van Dujin, M., Keijzer, F., \& Franken, D. (2006). Principles of Minimal Cognition. Casting Cognition as Sensorimotor Coordination. Journal of Adaptive Behavior, 14(2), 157-170.

Varela, F. J. (1979). Principles of biological autonomy. New York: North Holland. 
Varela, F.J., Thompson, E., \& Rosch, E. (1991). The Embodied Mind. Cognitive Science and Human Experience. Cambridge MA: MIT Press.

Von Uexküll, J. (1926). Theoretical Biology. New York: Harcourt, Brace \& Co.

Waddington, C. H. (1968). The basic ideas of biology. In C. H. Waddington (ed.), Towards a Theoretical Biology, Vol. I, Prolegomena, Chicago: Adline, 1-41.

Wadhams, G. H. \& Armitage, J. P. (2004). Making sense of it all: bacterial chemotaxis. Nature Reviews. Molecular Cell Biology, 5, 1024-1037.

Ziemke, T. \& Lowe, R. (2009). On the Role of Emotions in Embodied Cognitive Architectures: From Organisms to Robots. Cognitive Computation 1(1): 104-117. 